\title{
USO DE FUENTES DE INFORMACIÓN EN MÉDICOS RECIÉN GRADUADOS DE LIMA
}

\author{
Christian R. Mejia ${ }^{1, a}$, Onice J. Caceres ${ }^{2, b}$, Claudia A. Vera ${ }^{2, b}$, Ayar Nizama-Vía ${ }^{1, b ;}$, Walter H. Curioso \\ Percy Mayta-Tristán ${ }^{1, a}$, Red LIRHUS*
}

\begin{abstract}
RESUMEN
Con el objetivo de determinar el uso de fuentes de información en médicos recién graduados, de Lima, en abril del 2011, se realizó una encuesta a egresados de siete universidades, en quienes se indagó acerca del uso de buscadores del área de salud durante su año del internado médico; se definió como uso regular si manifestaban que usaron la fuente una vez por semana o a diario. En 490 encuestados las fuentes de información usadas regularmente fueron: SciELO, consultada por 173 (36,4\%); PubMed 165 (34,4\%); HINARI 117 (25,5\%); UpToDate 98 (22,3\%); Biblioteca Cochrane 94 (20,6\%); LILACS 91(19,8\%); biblioteca institucional hospitalaria 70 (15,0\%); BVS Perú 42 (9,3\%) y LIPECS $39(8,7 \%)$. Solo una minoría accedió regularmente a fuentes de información relacionadas con salud. Es necesaria mayor capacitación en el uso eficiente de los diversos recursos de información científica, de modo continuo y que llegue a los estudiantes y profesionales de la salud.
\end{abstract}

Palabras clave: Internet; Bibliografía; Bases de datos de citas; médicos, Perú (fuente: DeCS BIREME)

\section{USE OF INFORMATION SOURCES BY RECENTLY GRADUATED PHYSICIANS OF LIMA}

\begin{abstract}
In order to determine the use of information sources by recently graduated physicians of Lima, Peru in 2011, a survey was conducted among graduated physicians at seven universities. They were asked about the use of search engines in the health area during their year of medical internship [last year of medical school]. Regular use was defined as the source being used once a week or daily. For 490 respondents, regularly used information sources were SciELO, accessed by 173 (36.4\%); PubMed 165 (34.4\%); HINARI 117 (25.5\%); UpToDate 98 (22.3\%); Cochrane Library 94 (20.6\%); LILACS 91 (19.8\%); a hospital institutional library 70 (15.0\%); LIPECS 39 (8.7\%); and Peru BVS 42 (9.3\%). Only a minority regularly accessed information sources related to health. It is necessary to improve capacity in the efficient use of various resources of scientific information in a continuous way and that reaches students and health professionals.
\end{abstract}

Key words: Internet; Bibliografía; Citation databases; médicos, Perú (source: MeSH, NLM).

\section{INTRODUCCIÓN}

La información médica se encuentra en permanente y constante actualización, por lo que el médico contemporáneo necesita disponer y utilizar fuentes de información que cubran sus necesidades de nuevo conocimiento, que le permitan ejercer apropiadamente la medicina basada en evidencias y promover la educación médica continua (1). Dentro de estos recursos se encuentran las clásicas fuentes físicas (ej. libros y revistas impresas) y las nuevas herramientas electrónicas especializadas, como bases de datos de literatura científica y revistas electrónicas disponibles en internet ${ }^{(2-4)}$.

Todo profesional de la salud se ve en la necesidad de recurrir al conocimiento generado en el ámbito nacional e internacional, sin embargo, la investigación y producción científica local es todavía bastante limitada $(5,6)$. Si bien, internet es un medio frecuentemente usado

Escuela de Medicina, Universidad Peruana de Ciencias Aplicadas. Lima, Perú.

Sociedad Científica de Estudiantes de Medicina de la Universidad Ricardo Palma. Lima, Perú.

Dirección de Evaluación y Gestión del Conocimiento. Consejo Nacional de Ciencia, Tecnología e Innovación Tecnológica (CONCYTEC), Presidencia del Consejo de Ministros del Perú. Lima, Perú.

* Grupo Colaborativo Latinoamericano de Investigación en Recursos Humanos en Salud.

a Médico cirujano; ${ }^{\mathrm{b}}$ estudiante de Medicina; ${ }^{\mathrm{a}}$ doctor en Informática Biomédica

Recibido: 09-07-14 Aprobado: 05-11-14

Citar como: Mejia CR, Caceres OJ, Vera CA, Nizama-Vía A, Curioso WH, Mayta-Tristán P, et al. Uso de fuentes de información en médicos recién graduados de Lima. Rev Peru Med Exp Salud Publica. 2014;31(4):716-20. 
para la búsqueda de información ${ }^{(7)}$, son escasas las investigaciones locales que muestran el uso de tecnologías y fuentes de la información en médicos peruanos, existiendo algunos reportes en especialidades médicas de determinadas instituciones de salud ${ }^{(8,9)}$. Por lo expuesto, nuestro objetivo fue el determinar el uso de fuentes de información en médicos recién graduados de Lima.

\section{EL ESTUDIO}

Estudio observacional de corte transversal durante el mes de abril de 2011, en las instalaciones del Colegio Médico del Perú (CMP). El muestreo fue no probabilístico de tipo censal, se incluyó a los médicos que estuvieron inscritos para realizar el curso de inducción al Servicio Rural y Urbano Marginal en Salud (SERUMS) en el CMP, tomándose en cuenta a los egresados de alguna universidad de Lima Metropolitana y que voluntariamente aceptaron participar del estudio, se excluyó a los que no completaron la variable de interés, que no habían realizado el internado el año previo a la toma de datos 0 que no lo especificaron.

Se preguntó acerca del uso de bases de datos durante el año del internado relacionadas con la salud -buscador físico o electrónico que permite acceder a fuentes de información de tipo científica- (biblioteca institucional hospitalaria, PubMed, Biblioteca Cochrane, UpToDate, HINARI, SciELO, LILACS, LIPECS y BVS Perú) ${ }^{(10)}$, cada opción tuvo seis posibles respuestas $(0=$ no conoce; $1=$ nunca lo usó; $2=$ =lo usó una vez al año; 3=lo usó una vez al mes; 4=lo usó una vez a la semana, y 5=lo usó a diario). Se definió como uso regular si manifestaron que usaron la fuente una vez por semana o a diario. También se consignaron variables socioeducativas (edad, sexo, estado civil, universidad de procedencia, hospital donde realizó el internado, manejo del idioma inglés, presentación de trabajo de investigación en el pregrado, titulación por la modalidad de tesis y publicación de investigación científica), la frecuencia de uso de internet, uso de claves de bases de datos no personales, uso de la red social Facebook, características de los medios para acceder a la información (tener computadora o internet en casa, computadora portátil, internet inalámbrico y smartphone) y el uso de bases de datos.

El equipo investigador recogió, revisó y asignó un código a las encuestas, aquellas que no fueron completadas de manera adecuada fueron excluidas. Luego se procedió a la doble digitación en el programa Microsoft Excel versión 2010 (Microsoft Corporation, CA, USA). Se realizó el análisis de datos en el programa Stata 11,1 (Stata Corporation, TXT, USA). Para el análisis descriptivo de las variables numéricas se evaluó los supuestos de normalidad usando la prueba de Shapiro Wilk, según eso se describió la mejor medida de tendencia central y dispersión, para las variables categóricas se describieron las frecuencias y porcentajes. El proyecto fue aprobado por el Comité de Ética en Investigación del Instituto Nacional de Salud del Perú y se obtuvo la autorización del Decanato del Colegio Médico del Perú para la ejecución del estudio. Se invitó a los médicos inscritos a participar del estudio, se les proporcionó la encuesta, la cual fue autoaplicada. Se dio a conocer el objetivo del estudio y su importancia, se obtuvo el consentimiento verbal de los sujetos de investigación para participar del estudio. La encuesta fue anónima y los datos fueron confidenciales.

\section{HALLAZGOS}

La mediana de la edad de los 490 participantes fue 25 años (rango: 22-39 años); 297 (60,6\%) fueron mujeres; $471(96,1 \%)$ fueron solteros; $181(37,4 \%)$ egresaron de la Universidad San Martín de Porres; 99 (20,4\%) de la Universidad Ricardo Palma; 69 (14,3\%) de la Universidad San Juan Bautista; 45 (9,3\%) de la Universidad Científica del Sur; $39(8,1 \%)$ de la Universidad Nacional Federico Villarreal; $36(7,4 \%)$ de la Universidad Nacional Mayor de San Marcos y $15(3,1 \%)$ de la Universidad Peruana Cayetano Heredia; $349(71,8 \%)$ refirieron haber realizado su internado médico en un hospital del Ministerio de Salud; 76 (15,6\%) en algún hospital de las Fuerzas Armadas o Policía Nacional; 51 (10,5\%) en un hospital de la Seguridad Social (EsSalud) y $10(2,1 \%)$ en clínicas privadas.

Las características académicas hasta la culminación del pregrado fueron: $135(30,7 \%)$ manifestaron tener un dominio avanzado del inglés; $49(10,1 \%)$ presentaron un trabajo de investigación en algún congreso científico; $48(9,8 \%)$ se titularon por la modalidad de sustentación de tesis y $44(9,1 \%)$ publicaron alguna investigación. En cuanto a las características del uso de internet, 486 $(99,8 \%)$ manifestaron el uso regular del servicio de internet, $10 \mathrm{~h}$ fue la mediana de uso semanal (rango: 0,5151 h) y $237(52,0 \%)$ manifestaron que poseían claves de acceso no personales a páginas de información médica; $462(95,1 \%)$ manifestaron que usaban la red social Facebook, de ellos, $226(49,3 \%)$ accedían más de una vez al día; 149 (32,6\%) una vez al día; 61 $(13,3 \%)$ más de una vez a la semana; $20(4,4 \%)$ una vez a la semana y $2(0,4 \%)$ una vez o más al mes. Las características de los medios para acceder a internet se muestran en la Tabla 1.

Las fuentes de información usadas regularmente fueron: SciELO, accedida por $173(36,4 \%)$ encuestados, seguida por PubMed 165 (34,4\%), HINARI 117 (25,5\%), UpToDate 
Tabla 1. Características de los medios para acceder a Internet en egresados de facultades de Medicina de Lima, 2011

\begin{tabular}{lc}
\hline \multicolumn{1}{c}{ Medios de acceso a internet } & N (\%) \\
\hline Tiene computadora en casa & \\
Sí & $460(96,2)$ \\
No & $18(3,8)$ \\
Tiene internet en casa & \\
Sí & $453(95,2)$ \\
No & $23(4,8)$ \\
Tiene computadora portátil (laptop/netbook) \\
Sí & $390(81,9)$ \\
No & $86(18,1)$ \\
Tiene internet inalámbrico o portátil & \\
Sí & $335(75,1)$ \\
No & $111(24,9)$ \\
Tiene un teléfono Smartphone con internet \\
Sí & $103(24,6)$ \\
No & $315(75,4)$ \\
\hline
\end{tabular}

98 (22,3\%), Biblioteca Cochrane 94 (20,6\%), LILACS $91(19,8 \%)$, biblioteca institucional hospitalaria 70 (15,0\%), BVS Perú 42 (9,3\%) y LIPECS 39 (8,7\%). La frecuencia de uso de fuentes de información durante el año de internado de los egresados se muestra en la Tabla 2.

\section{DISCUSIÓN}

Se encontró que la frecuencia de uso de recursos de información disponibles en internet en una población de médicos recién graduados fue baja. Asimismo, aproximadamente un tercio de los encuestados solo visitó una vez la biblioteca física en su último año de carrera. La población encuestada en este estudio tiene características similares de uso de las tecnologías y fuentes de información al de otros grupos de nuestro medio, tanto de médicos como de estudiantes del pregrado (11). Este hallazgo se podría explicar por diversos factores. El primero es que los encuestados no pudieron acceder a las fuentes de información por la limitada accesibilidad y/o logística inadecuada. Sin embargo, este factor es poco probable en esta población, ya que la gran mayoría accedía a internet a diario, sobre todo para el uso de las redes sociales, así mismo, la mayoría contaba con acceso remoto para poder acceder a recursos electrónicos. Casi un tercio de la población contaba con un smartphone con internet, lo que sugiere que los dispositivos móviles son cada vez más usados, y deben ser promovidos como otra herramienta para el acceso remoto a fuentes de información disponibles en internet, como lo demuestran estudios internacionales ${ }^{(12)}$, ya que permiten poder acceder con mayor facilidad a la información de bases de datos electrónicas, pudiéndolo hacer incluso justo en el momento que se genera la duda, durante la atención del paciente ${ }^{(13,14)}$.

Adicionalmente, la falta de dominio del idioma Inglés puede ser un desafío adicional que limite una eficiente búsqueda en fuentes de información, ya que la vasta información relacionada con salud en su mayoría está disponible y publicada en inglés ${ }^{(15,16)}$. Otros factores que podrían explicar lo hallado es que los recién graduados no tienen el conocimiento o las habilidades necesarias para poder utilizar eficientemente las fuentes de información electrónicas, o que los alumnos no encuentren utilidad o no vean como una necesidad el buscar información por estos medios, lo cual no fue evaluado en este estudio. Es necesario realizar estudios que permitan caracterizar mejor estos posibles factores.

Al evaluar el perfil científico que tenían los encuestados, se pudo observar que muy pocos tuvieron experiencia científica en el pregrado, ya que, solo uno de cada diez se había titulado por tesis, había presentado un trabajo de investigación en un evento científico o había publicado en una revista indizada. Es necesario evaluar en futuros estudios diversas estrategias de capacitación en investigación y redacción de trabajos científicos a nivel de pregrado y posgrado, que permitan incrementar el número de publicaciones ${ }^{(17-19)}$.

Una de las competencias importantes que deben tener los profesionales de la salud es ser autodidactas para su formación continua, por lo que las universidades

Tabla 2. Uso de fuentes de información en graduados de facultades de Medicina de Lima, 2011

\begin{tabular}{lcccccc}
\hline \multirow{2}{*}{ Fuente } & \multicolumn{5}{c}{ Frecuencia de uso de la fuente de información (\%) } \\
\cline { 2 - 7 } \multicolumn{1}{c}{} & No la conozco & Nunca la uso & Una por año & Una por mes & Una por semana & Todos los días \\
\hline PubMed & $13(2,7)$ & $39(8,1)$ & $106(22,0)$ & $158(32,8)$ & $152(31,7)$ & $13(2,7)$ \\
Biblioteca Cochrane & $22(4,8)$ & $94(20,5)$ & $100(21,8)$ & $148(32,3)$ & $88(19,3)$ & $6(1,3)$ \\
UpToDate & $53(12,0)$ & $138(31,4)$ & $72(16,4)$ & $79(17,9)$ & $72(16,4)$ & $26(5,9)$ \\
SciELO & $15(3,2)$ & $31(6,5)$ & $86(18,1)$ & $170(35,8)$ & $147(30,9)$ & $26(5,5)$ \\
HINARI & $16(3,5)$ & $73(15,9)$ & $111(24,2)$ & $142(30,9)$ & $104(22,7)$ & $13(2,8)$ \\
LILACS & $32(7,0)$ & $114(24,8)$ & $100(21,8)$ & $122(26,6)$ & $81(17,6)$ & $10(2,2)$ \\
BVS Perú & $77(17,1)$ & $182(40,5)$ & $62(13,8)$ & $87(19,3)$ & $37(8,2)$ & $5(1,1)$ \\
LIPECS & $95(21,2)$ & $193(43,2)$ & $63(14,1)$ & $57(12,8)$ & $33(7,4)$ & $6(1,3)$ \\
Biblioteca del hospital & $35(7,5)$ & $112(24,0)$ & $141(30,3)$ & $108(23,2)$ & $67(14,4)$ & $3(0,6)$ \\
\hline
\end{tabular}

BVS: Biblioteca virtual en salud. 
deben asegurar la formación continua y evaluaciones en gestión de recursos de la información de sus egresados. Esto cobra más importancia ahora, ya que todas las universidades tienen que asegurarse que sus estudiantes adquieran estas capacidades para cumplir con el requisito de obligatoriedad de la titulación por tesis, que es uno de los cambios de la nueva ley universitaria ${ }^{(20)}$. Sin embargo, consideramos que este deficiente uso disminuirá debido al creciente acceso a bases de datos científicas que actualmente brindan universidades e incluso el Consejo Nacional de Ciencia, Tecnología e Innovación Tecnológica, a través del Repositorio Nacional Digital de Acceso Abierto, que concentra y preserva la producción en ciencia, tecnología e innovación del Perú (http://alicia.concytec. gob.pe). Además de la suscripción a bases de datos como ScienceDirect Freedom Collection y SCOPUS, por parte de universidades, institutos de investigación a nivel nacional y a aquellas personas registradas en el Directorio Nacional de Investigadores (http://directorio. concytec.gob.pe).

Se tienen algunas limitaciones en el estudio. Primero, la población evaluada no representa, necesariamente a los médicos peruanos que acaban de egresar, debido a que este estudio incluyó solo a un grupo médicos recién graduados de Lima. Sin embargo, los resultados obtenidos son muy útiles, ya que reflejan el uso de bases de datos de una gran cantidad de médicos que tenían la intención de realizar su práctica asistencial en la atención primaria. Además, el sesgo de memoria pudo estar presente, ya que se preguntó a los egresados después de tres meses de culminado el internado, pero se cree que esto no influyó en gran medida, ya que se excluyó a los que no habían realizado el internado recientemente y se consultó por actividades que las realizaron durante todo un año. No se evaluó estrategias de búsqueda, tampoco patrones de uso o el uso de los metabuscadores (Google o Google académico), ya que no fue objetivo del estudio, esto debería ser evaluado en otras investigaciones que indaguen de una mayor cantidad de recursos y/o el uso de los artículos en sí.

En conclusión, en base a los datos analizados, los recién graduados de la carrera de medicina en Lima tuvieron una baja frecuencia de uso de fuentes de información como estrategia de búsqueda. Se recomienda que se evalúe las estrategias que utilizan los estudiantes para acceder a fuentes científicas. Es necesaria mayor capacitación dirigida a usar eficientemente los diversos recursos de información relacionados con salud, y de manera continua, en estudiantes de medicina y profesionales de la salud. Los resultados de este estudio tienen implicancias para elaborar estrategias óptimas de difusión de las bases de datos de literatura científica en salud, incluyendo capacitaciones virtuales.

Contribuciones de autoría: CRM, ANV, WHC, CAV y PMT tuvieron la idea de investigación y diseñaron el estudio, CRM recopilaron los datos, CRM, OJC, CAV realizaron el análisis e interpretación de datos, todos los autores participaron en la redacción del manuscrito y aprobaron la versión final a publicar.

Miembros de Red LIRHUS: Reneé Pereyra-Elías, Edén Galán-Rodas.

Fuentes de financiamiento: Autofinanciado.

Conflictos de interés: Los autores declaran no tener conflictos de interés.

\section{REFERENCIAS BIBLIOGRÁFICAS}

1. Curran VR, Fleet LJ, Kirby F. A comparative evaluation of the effect of internet-based cme delivery format on satisfaction, knowledge and confidence. BMC Med Educ. 2010;10(10):1-7.

2. Castrillón-Estrada J, García J, Anaya M, Rodríguez D, De La Rosa D, CaballeroUribe C. Bases de datos, motores de búsqueda e índices temáticos: herramientas fundamentales para el ejercicio médico. Salud Uninorte. 2008;24(1):96-119.

3. Hiller S. Assessing user needs, satisfaction and library performance at the University of Washington Libraries. Library Trends. 2001;49(4):605-25.

4. Guerrero JC, Amell I, Cañedo R. Tecnología, tecnología médica y tecnología de la salud: algunas consideraciones básicas. Acimed. 2004;12(4):1-16.

5. Huamani C, Mayta-Tristán P. Producción científica peruana en medicina y redes de colaboración, análisis del Science Citation Index 2000-2009. Rev Peru Med Exp Salud Publica. 2010 Jul-Set;27(3):315-25.

6. Galán E, Manrique N, Villavicencio E, Yllatopa E, Peralta M, De la Cruz W. Producción científica de los investigadores del pregrado de medicina humana del Perú, 1993-2003. CIMEL. 2005;10(1):41-8.

7. Pelzer N, Wiese W, Leysen J. Library use and information seeking behaviour of veterinary medical students revised in the electronic environment. B Med Libr Assoc. 1998;86(3):346-55.
8. Canelo C, Alarcon J, Amao E, Beteta $\mathrm{V}$, Monge E. Conocimientos, actitudes y prácticas de la medicina basada en evidencias en médicos asistentes y residentes en dos hospitales de LimaPerú. Rev Med Hered. 2007 AbrJun;18(2):76-84.

9. Herrera-López V, Stucchi-Portocarrero $S$, Vega-Galdós F. Encuesta virtual sobre uso de Internet y medios informáticos entre psiquiatras y residentes de psiquiatría en el Perú. Rev Neuropsiquiatr. 2013;74(1):191-9.

10. Lai NM, Nalliah S. Informationseeking practices of senior medical students: the impact of an evidencebased medicine training programme. Educ Health (Abingdon). 2010 Apr;23(1):151. 
11. Angulo-Bazán Y, Borja-García R, Alegre T, Alva C, Gutiérrez G. Gestión de la información cientifica en estudiantes de medicina de una universidad pública peruana. CIMEL. 2010;15(1):23-7.

12. Batten LM, Chowdhury M, Drew J. The major drawbacks to the use of wireless communication products in education. 1st Australian Information Security Management Conference; Perth, Western Australia: AISMC; 2003.

13. Walton G, Childs S, Blenkinsopp E. Using mobile technologies to give health students access to learning resources in the UK community setting. Health Info Libr J. 2005 Dec;22 Suppl 2:51-65.

14. Horna P, Curioso W, Guillén C, Torres C, Kawano J. Conocimientos, habilidades y características del acceso a internet en estudiantes de medicina de una Universidad Peruana. An Fac Med. 2002;63(1):32-9.

15. Veloz-Martínez MG, Almanza-Velasco E, Uribe-Ravell JA, González LL, Quintana-Romero V, Alanís-López P. Uso de tecnologías en información y comunicación por médicos residentes de ginecología y obstetricia. Inv Ed Med. 2012;1(4):183-9.

16. Molina-Ordóñez J, Huamaní C, Mayta-Tristán P. Apreciación estudiantil sobre la capacitación universitaria en investigación: estudio preliminar. Rev Peru Med Exp Salud Publica. 2008;25(3):325-9.

17. Estrada JM. La búsqueda bibliográfica y su aplicación en PubMed-MEDLINE. SEMERGEN - Medicina de Familia. 2007;33(4):193-9.
18. Monteagudo JL. Tecnologías de la Información y Comunicaciones. Educ Med. 2004;7(1):15-22.

19. Arriola-Quiroz I, Curioso WH, Cruz-Encarnacion M, Gayoso O. Characteristics and publication patterns of theses from a Peruvian medical school. Health Info Libr J. 2010 Jun;27(2):148-54. doi: 10.1111/j.1471-1842.2010.00878.x

20. Perú, Congreso de la República. Decreto Ley $N^{\circ}$ 30220: Ley Universitaria. Lima: Congreso de la República; 2014.

Correspondencia: Christian Mejia.

Dirección: Av. Las Palmeras 5713 - Los Olivos. Lima 39 - Perú.

Teléfono: (511) 997643516

Correo electrónico:christian.mejïa.md@gmail.com

\section{Consulte las ediciones anteriores de la Revista Peruana de Medicina Experimental y Salud Pública en} www.scielosp.org

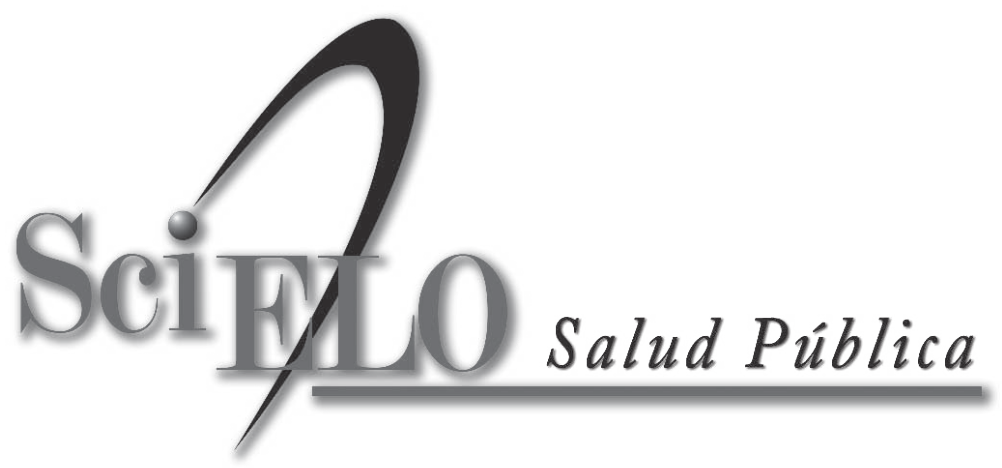

